\title{
A Model of Tracer Transport in Airway Surface Liquid
}

\author{
D.J. Smith*, E.A. Gaffney, J.R. Blake \\ School of Mathematics, University of Birmingham, Birmingham B15 2TT, UK
}

Received: 8 February 2006 / Accepted: 30 August 2006 / Published online: 18 January 2007 (C) Society for Mathematical Biology 2007

\begin{abstract}
This study is concerned with reconciling theoretical modelling of the fluid flow in the airway surface liquid with experimental visualisation of tracer transport in human airway epithelial cultures. The airways are covered by a dense mat of cilia of length $\sim 6 \mu \mathrm{m}$ beating in a watery periciliary liquid (PCL). Above this there is a layer of viscoelastic mucus which traps inhaled pathogens. Cilia propel mucus along the airway towards the trachea and mouth. Theoretical analyses of the beat cycle (Smith et al., 2006b; Fulford and Blake, 1986) predict small transport of PCL compared with mucus, based on the assumption that the epithelium is impermeable to fluid. However, an experimental study (Matsui et al., 1998) indicates nearly equal transport of PCL and mucus. Building on existing understanding of steady advection-diffusion in the ASL (Blake and Gaffney, 2001; Mitran, 2004), numerical simulation of an advection-diffusion model of tracer transport is used to test several proposed flow profiles and to test the importance of oscillatory shearing caused by the beating cilia. A mechanically derived oscillatory flow with very low mean transport of PCL results in relatively little 'smearing' of the tracer pulses. Other effects such as mixing between the PCL and mucus, and significant transport in the upper part of the PCL above the cilia tips are tested and result in still closer transport, with separation between the tracer pulses in the two layers being less than $9 \%$. Furthermore, experimental results may be replicated to a very high degree of accuracy if mean transport of PCL is only $50 \%$ of mucus transport, significantly less than the mean PCL transport first inferred on the basis of experimental results.
\end{abstract}

Keywords Mucus · Cilia · Tonicity · Advection · Diffusion · Dispersion

\section{Introduction}

The airways of the lung are lined with a thin $(\sim 5-20 \mu \mathrm{m})$ protective layer of liquid which is secreted by the airway epithelium, then propelled towards the trachea and mouth by the action of cilia. The cilia beat in a watery periciliary liquid (PCL),

\footnotetext{
* Corresponding author.

E-mail address: smithd@for.mat.bham.ac.uk (D.J. Smith).
} 
above which lies a viscoelastic mucous layer consisting of water and glycosylated mucin proteins. There has been considerable doubt regarding the mean axial transport of PCL, since theoretical studies (Smith et al., 2006b; Fulford and Blake, 1986) have predicted that transport is small compared with transport of mucus, whereas experimental studies using tracer particles in cilia cultures (Matsui et al., 1998) appeared to show equal transport (cotransport) of mucus and PCL. This paper is concerned with reconciling theoretical results and experimental observations, and in particular we seek to discover what mean transport profiles may be consistent with the observed tracer transport results. The modelling approach has applications to similar problems, for instance morphogen transport causing symmetry breaking in the embryonic ventral node (see for example Okada et al., 2005).

In this study we briefly describe the characteristics of the muco-ciliary system in the lungs, then discuss the question regarding the normal tonicity of airway surface liquid (ASL) and the related issue of the mean transport of periciliary liquid (PCL). We describe the contradictory conclusions arising from in vitro experiments of Matsui et al. (1998) and the theoretical modelling work of Smith et al. (2006b) and Fulford and Blake (1986). An existing physically-based phenomenological model of the time dependent fluid flow in the ASL (Smith et al., 2006b) based on the assumption that the epithelium is impermeable to fluid is briefly reviewed. Tracer transport and dispersion under this, and other suggested velocity profiles, are modelled as an advection-diffusion process similar to that of Blake and Gaffney (2001). The resulting equations are solved using an alternating direction implicit (ADI) method, together with the QUICK scheme of Leonard (1979) for advective discretisation. Numerical results are interpreted quantitatively, leading to insight into the apparent conflict between experimental results and theoretical modelling.

\subsection{Muco-ciliary clearance}

The airway epithelium is covered with a dense mat of cilia, of length $\sim 5-7 \mu \mathrm{m}$, radius $\sim 0.1 \mu \mathrm{m}$ and spaced around $0.3-0.4 \mu \mathrm{m}$ apart (data from Sleigh et al., 1988; Toskala, 1994). The cilia beat in coordinated waves with a frequency of around 6-15 Hz. The ciliary beat is asymmetric, consisting of an 'effective' or propulsive stroke, and a 'recovery stroke', the effective stroke lasting about a fifth as long as the entire beat (Sanderson and Sleigh, 1981). During the effective stroke the cilium is fully extended so that it penetrates the overlying mucous layer, propelling it along the airway towards the trachea and mouth. During the recovery stroke, the cilium bends slightly so that it does not engage the mucous layer.

Detailed modelling of the fluid dynamics of muco-ciliary flow (Smith et al., 2006b; Fulford and Blake, 1986) predicts that the mean transport of PCL is very small in comparison to transport of mucus. Such studies assume that the epithelium is impermeable to fluid, consistent with the hypotonic ASL hypothesis described below, and indeed due to the converging geometry of the airways, if no fluid is absorbed by the epithelium, the PCL thickness would not be maintained at the depth of $\sim 5 \mu \mathrm{m}$ that is essential for efficient coupling of the cilia and mucus. A typical mean profile reproduced from Smith et al. (2006b) is given in Fig. 6. 


\subsection{Salt/fluid controversy and cystic fibrosis}

Accurate understanding of muco-ciliary clearance is important in that it may help to improve the understanding of the pathogenesis and treatment of respiratory disease-in particular cystic fibrosis (CF) lung disease. $\mathrm{CF}$ is a disorder arising from a mutation in the cystic fibrosis transmembrane regulator (CFTR), one of the symptoms of which is chronic bacterial infection of the lung. Various explanations have been advanced for the pathogenesis of $\mathrm{CF}$, we shall be concerned with those relating to abnormal ion transport by CFTR, and how the ASL is regulated by the epithelium.

The 'hypotonic ASL' hypothesis (described by Smith et al., 1996) postulates that normal epithelia are covered by an ASL with sufficiently low salt concentration (hypotonicity) to activate defensins and create an antimicrobial 'shield' on airway surfaces. The epithelium extracts salt, but not water, from the PCL. In CF patients, salt is not extracted due to a defect in $\mathrm{Cl}^{-}$conductance, rendering defensins inactive. Implicit in this hypothesis (Quinton, 1994) is that PCL is not transported along with the mucus out of the lungs. This must be the case because the surface area of the lungs decreases greatly moving from the bronchioles to the bronchia and finally the trachea, but the ASL depth does not greatly increase.

The 'isotonic volume' hypothesis (Boucher, 1994) predicts that airway epithelia regulate the volume of the ASL by isotonic ion and water transport to optimise mucus clearance. In CF the rate of ion and water transport is abnormally high, reducing PCL volume, concentrating and dehydrating mucus, resulting in plugging of the airways and infection. This is consistent with the idea that there is significant PCL transport along the converging airways. If this hypothesis is true, novel treatments for CF should focus on restoring ASL volume (Matsui et al., 1998) rather than changing ASL tonicity.

In Section 2, we briefly summarize the experiments of Matsui et al. (1998), then in Section 3 we describe the theoretical modelling framework of Smith et al. (2006b).

\section{Tracer dispersion experiments of Matsui et al. (1998)}

Below we briefly summarise the experimental study carried out by Matsui et al. (1998). In suitable media the cultures produced a mucous layer around $25 \mu \mathrm{m}$ in depth and in around a quarter of cases exhibited spontaneous circular transport. Amongst other techniques, the ASL was labelled with caged fluorescein-dextran, which could be photoactivated in $400 \mu \mathrm{m}$ columns. It was found that over periods of around $20 \mathrm{~s}$, the photoactivated regions moved at the same speed in both the PCL and mucous layer. Average circular transport speeds of $39.2 \mu \mathrm{m} / \mathrm{s}$ were reported, with higher values at the outer edge of the rotating mucus. Matsui et al. (1998) shows a fluorescent region before and after $20 \mathrm{~s}$. There is no smearing between the two images, as would be expected if the PCL had not been transported along with the mucus (Matsui et al., 1998). Confocal microscopy allowed vertical sections of ASL to be visualised. There was no difference in transport of fluorescent particles with height above the epithelium. This technique was used to 
A

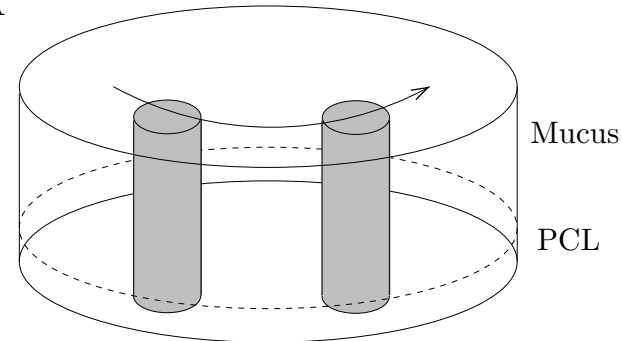

B

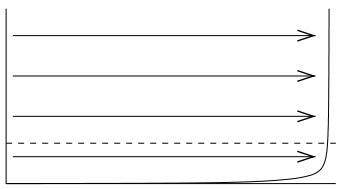

Fluid velocity

Fig. $1 \mathrm{~A}-\mathrm{a}$ simplified representation of the cotransport phenomenon found by Matsui et al. (1998). B-a schematic of the observed profile of Matsui et al. (1998).

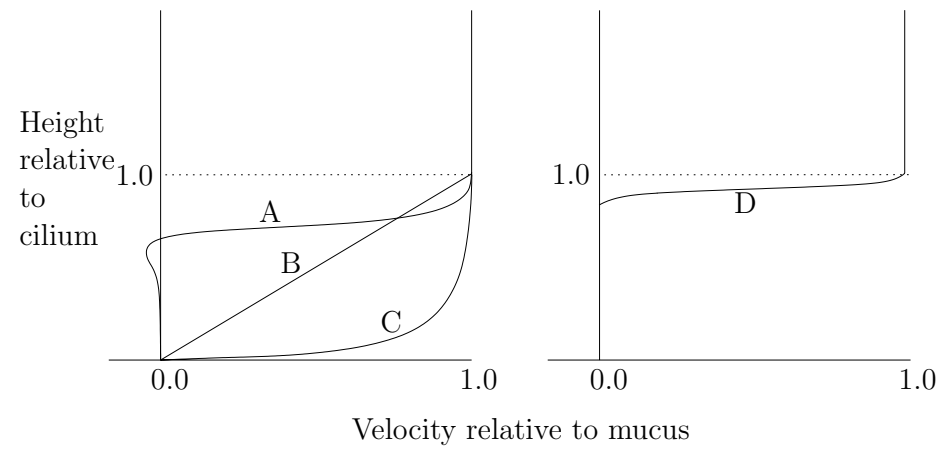

Fig. 2 Comparing the velocity profiles predicted by theory (typified by A), shear driven (plane Couette) flow (B) and those apparently found by experiment (C)-redrawn from Matsui et al. (1998). Graph (D) shows a mean profile from the model of Smith et al. (2006b).

estimate the diffusion coefficients of dextran in mucus and PCL: $3.6 \mu \mathrm{m}^{2} \mathrm{~s}^{-1}$ and $160 \mu \mathrm{m}^{2} \mathrm{~s}^{-1}$ respectively. Diffusion coefficients were measured in non cilio-active cultures, and so represent spreading of tracer due to diffusion only and not due to transport by cilia.

At first sight the results of Matsui et al. (1998) appear to show that tracer particles in the PCL are advected along at the same speed as tracer particles in the mucous layer, implying that the fluid velocity in both layers is approximately equal. However, diffusion of tracer between the two layers must also be taken into account: in Blake and Gaffney (2001) it was shown that even if fluid transport in the PCL is two orders of magnitude smaller than in the mucous layer, tracer transport in the PCL may be at least half that in the mucous layer. This is essentially the phenomenon known as 'Taylor Disperson' (Taylor, 1953). Further simulation using various steady advection profiles by the UNC Virtual Lung Group (Mitran, 2004) confirms this, together with showing the effect of the varying the diffusion coefficient in the PCL.

A measure of the relative effect of advective and diffusive transport is the Peclet number $U L / D$, where $U, L$ and $D$ are typical values for velocity, length and the diffusion coefficient. Since transport is approximately $40 \mu \mathrm{m} / \mathrm{s}$ and each layer is 
approximately $6 \mu \mathrm{m}$ thick, the Peclet numbers are 1.5 in the PCL and 67 in the mucous layer. This suggests that in the PCL, diffusion and advection will be approximately equally important, with diffusion tending to obliterate gradients in tracer transport caused by advection. In the mucous layer advection will dominate, and the large gradients in advective transport near the interface will be preserved. However, the presence of oscillations in the flow caused by the beating cilia may be important. In the next section we describe briefly a recent model which quantifies these oscillations.

\section{The traction layer model-a physically-based phenomenological model of fluid transport}

The model, described in full in Smith et al. (2006b), considers most of the relevant features of the muco-ciliary system-metachronism, the spatial and temporal asymmetry of the beat cycle, the viscous resistance of the cilia sublayer, surface and interface tension and the viscoelasticity of the mucous layer. Unlike much previous modelling this predicts the time dependent oscillations in the flow field in addition to the mean transport. It does not feature osmotic fluxes through the epithelium, and so does not model the isotonic volume hypothesis discussed above. The model is based upon the Stokes flow equations for very low Reynolds number flow $\mathbf{u}$ :

$$
\begin{array}{r}
\frac{\partial \sigma_{j k}}{\partial x_{k}}+f_{j}=0, \\
\nabla \cdot \mathbf{u}=0,
\end{array}
$$

where $f_{j}$ is a volume force, $\sigma_{j k}$ is the stress tensor and there is summation over the index $k=1,2,3$. Based on the observation (Sanderson and Sleigh, 1981) that the mucus surface and interface remain remarkably flat, the domain is divided into three layers, the Newtonian PCL $(0<z<h)$, the viscoelastic mucous 'traction layer' $(h<z<L)$ in which the cilia penetrate and propel the mucus, and the viscoelastic free mucous layer $(L<z<H)$ which is not subject to any volume force as the cilia are not present in this layer. Since lung cilia tend to be coordinated in the direction perpendicular to the transport direction the flow is taken to be constant in the $y\left(x_{2}\right)$ direction, so that the model is two dimensional, in the $(x, 0, z)$ plane. This is shown in Fig. 3. The PCL is taken to have viscosity $\mu^{P} \approx 0.001 \mathrm{Pas}$, the remaining layers have viscosity values $\mu^{M 1}$ and $\mu^{M 2}$ which are, in general, of the order of 5-50 times greater. The mucous layers are modelled as Maxwell fluids with relaxation time $\lambda_{1} \sim 0.03 \mathrm{~s}$. The values for $\mu^{M 2}$ and $\lambda_{1}$ are chosen by fitting to the data of Lutz et al. (1973) for canine tracheal mucus, the value of $\mu^{M 1}$ can be varied from $\mu^{P}$ up to $\mu^{M 2}$ in order to simulate a shear thinning effect due to the high shear rate in the penetration region.

In the PCL, the volume force $(f, 0, g)$ is of the form

$$
\begin{array}{ll}
f(x, z, t)=-\gamma_{x}^{P}\left(u-u_{\text {cilia }}\right), & 0<z<h, \\
g(x, z, t)=-\gamma_{z}^{P}\left(v-v_{\text {cilia }}\right), & 0<z<h,
\end{array}
$$




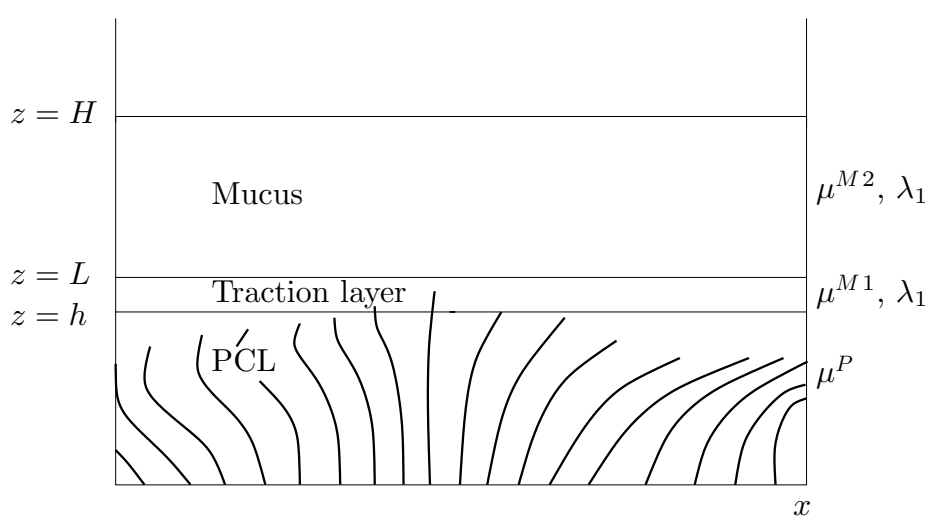

Fig. 3 Diagram of the 3 layer model. Newtonian PCL, viscosity $\mu^{P}$, depth $h$. Traction layer, viscosity $\mu^{M 1}$, elasticity $\lambda_{1}$, depth $L-h$. Mucous layer, viscosity $\mu^{M 2}$, elasticity $\lambda_{1}$, depth $H-L$.

where $\mathbf{u}_{\text {cilia }}$ is a Fourier series representation of the motion of the cilia sublayer and $\gamma_{x}^{P}, \gamma_{z}^{P}$ are resistance coefficients derived by considering the PCL as an active porous medium. In the traction layer, the force is of the form

$$
\begin{aligned}
& f(x, z, t)=-\left(\frac{1}{2} f_{0}+\sum_{n=1}^{15} f_{n} \cos [(k x+\sigma t) n]\right) \gamma_{x}^{M 1} U_{\mathrm{int}} \frac{\sin (\pi z / L)}{\sin (\pi h / L)}, \\
& g(x, z, t)=-\left(\sum_{n=1}^{15} g_{n} \sin [(k x+\sigma t) n]\right) \gamma_{z}^{M 1} V_{\mathrm{int}} \frac{\sin (\pi z / L)}{\sin (\pi h / L)} .
\end{aligned}
$$

Briefly, these were derived by introducing Fourier series $\sum f_{n}$ and $\sum g_{n}$ which define forward propulsion for one fifth of the beat cycle. The parameters $\sigma$ and $\kappa$ are the radian frequency and wavenumber, taking values of around $60 \mathrm{rad} / \mathrm{s}$ and $\pi / 15 \mu \mathrm{m}^{-1}$ respectively. The constants $U_{\text {int }}$ and $V_{\text {int }}$ represent the relative velocity of the fluid and cilium at $z=h$ during the midpoint of penetration, and the $\sin (\pi z / L)$ terms represent a monotonic decay of the force moving from $z=h$ to $z=L$. A full derivation is given in Smith et al. (2006b).

For boundary conditions we take no-slip and no-flux conditions on $z=0$, zero vertical velocity and continuity of tangential stress on $z=h$; continuity of velocity, normal and tangential stress on $z=L$; and zero vertical velocity and zero tangential stress on $z=H$. By Fourier series analysis, after some manipulation the problem is converted to a system of 30 first order ODEs for each term $\mathbf{u}_{n}$ which are solved numerically by a finite difference algorithm. The steady solution may be found analytically, and is of the form shown in Fig. 2(D). It is qualitatively similar to the discrete sublayer results of Fulford and Blake (1986), although with less flux in the upper part of the PCL, between $z=0.6 L$ and $z=h$. The mean mucus transport rate is found to be around $40 \mu \mathrm{m} / \mathrm{s}$, similar to the $39.2 \mu \mathrm{m} / \mathrm{s}$ found by Matsui et al. (1998). The full time dependent results, shown in Fig. 4 show oscillations of the order of nearly 10 times the mean flow. A detailed description of the results 
A

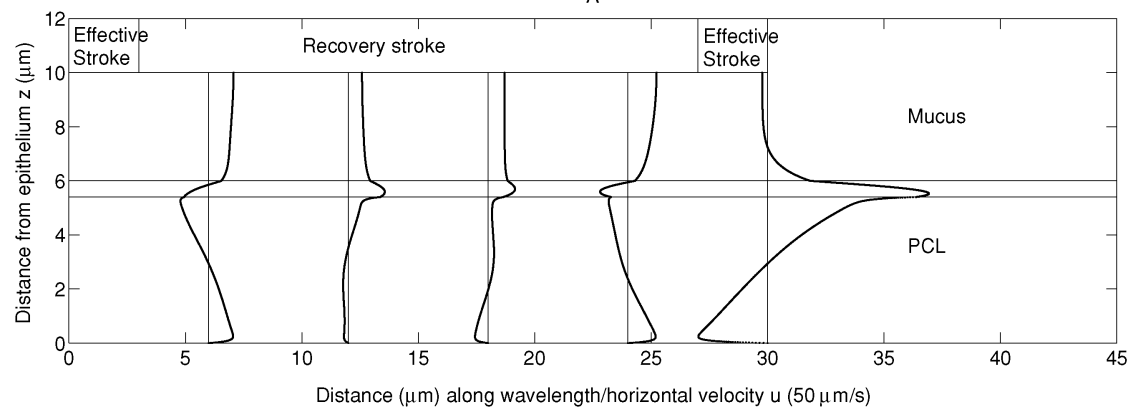

B

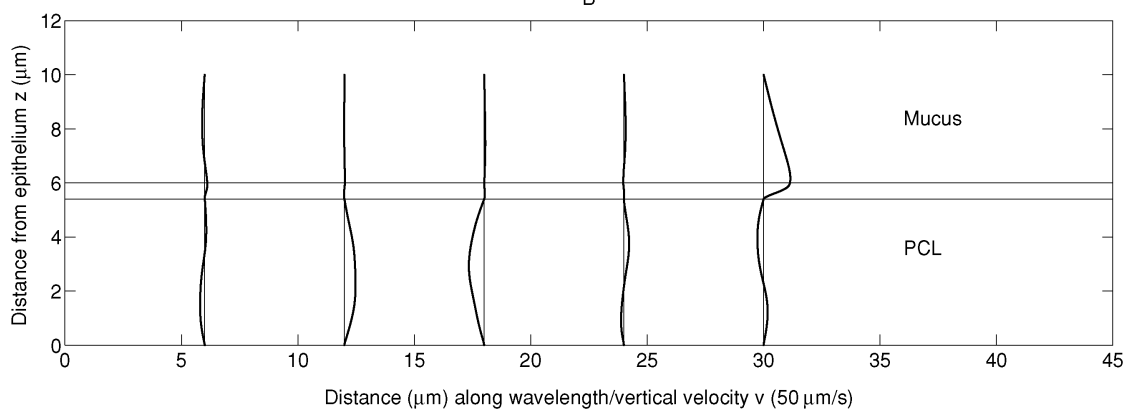

Fig. 4 Traction layer oscillatory velocity profiles used for tracer dispersion simulation: Ahorizontal velocity profiles at five points along the wavelength versus distance from epithelium. B-vertical velocity profiles at five points along the wavelength versus distance from epithelium. Parameter values: $L=6 \mu \mathrm{m}, \lambda=30 \mu \mathrm{m}, H=10 \mu \mathrm{m}, h=5.4 \mu \mathrm{m}, \sigma=60 \mathrm{rad} / \mathrm{s}, \lambda_{1}=0.03 \mathrm{~s}$. Viscosity ratios $\mu^{M 1} / \mu^{P}=6, \mu^{M 2} / \mu^{M 1}=8$. Sublayer resistance coefficient $\gamma_{x}=90^{2} \mu / L^{2}, \gamma_{z}=$ $75^{2} \mu / L^{2}$.

for different parameter sets, together with comparisons with studies of disease and drug effects is given in Smith et al. (2006b).

As can be seen from Fig. 2, the mean profiles predicted by theory and experiment are completely at variance. In an initial investigation of this paradox, Blake and Gaffney (2001) modelled tracer dispersion in ASL. Using a steady velocity profile typical of those found in Fulford and Blake (1986), they formulated an advection-diffusion equation and solved it using an ADI method, with first order upwind discretisation for the advective terms. It was found that the results were not consistent with the experimental results of Matsui et al., giving differential transport in the two layers. The simulation was repeated with a large ad hoc oscillatory component, indicating how oscillatory effects could produce cotransport. The discrepancy in transport between the two layers was still significant though reduced. In this study we extend this work, but using a more accurate numerical scheme which does not introduce numerical diffusion, and using a physically based oscillatory velocity field from Smith et al. (2006b). We shall investigate various explanations for the observed results, although we do not yet explicitly model osmotic flow. Furthermore, we use a quantitative measure of separation of the tracer pulses in order to assist with analysis of the results. In the following sections we describe a two dimensional advection-diffusion model of tracer transport, the 
numerical solution method, then results with various steady and oscillatory profiles including that of Smith et al. (2006b).

\section{Two dimensional advection-diffusion model}

The fluid transport model of Smith et al. (2006b) is two dimensional, in that flow and variations in the flow in the $x_{2}$ or $y$ direction are neglected. Due to the fact that the cilia are assumed to beat in phase in this direction, this is an acceptable approximation, although oscillations in this direction may enhance mixing slightly. We shall use a two dimensional model of tracer dispersion in the $(x, 0, z)$ plane for reasons of practicality - an additional spatial dimension would make computation orders of magnitude more expensive. Using $c(\mathbf{x}, t)$ to denote concentration of tracer, in the presence of a fluid flow field $\mathbf{u}$, the advective flux of tracer is $c \mathbf{u}$. By Fick's law, the diffusive flux will be $-D \nabla c$. Hence the total flux of tracer will be given by $\mathbf{J}=c \mathbf{u}-D \nabla c$. It is possible that other effects besides advection and diffusion may be present in the system, such as adhesion of tracer particles to the mucus, but these are beyond the scope of this study.

From the above modelling we have some insight into how diffusion acts to even out vertical gradients in the tracer profile caused by advection. The vertical component of the diffusive flux is $-D \partial c / \partial z$. As the pulses begin to separate over a very short vertical lengthscale, the gradient $\partial c / \partial z$ becomes large and hence the diffusive term may balance the advective horizontal flux $c u$.

Using conservation of tracer and the divergence theorem, we can derive

$$
\frac{\partial c}{\partial t}=\nabla \cdot(D \nabla c-c \mathbf{u})
$$

The diffusion coefficient $D$ will depend only on the properties of the fluid. Since the PCL occupies $0<z<h$ and the mucous layer $h<z<H$ we have $D=D(z)$. From incompressibility, the velocity field $u$ satisfies $\nabla \cdot \mathbf{u}=0$, leading to the simplification

$$
\frac{\partial c}{\partial t}=D \nabla^{2} c+\frac{\mathrm{d} D}{\mathrm{~d} z} \frac{\partial c}{\partial z}-\mathbf{u} \cdot \nabla c
$$

As in Blake and Gaffney (2001) we choose for initial conditions a rounded 'pulse' of photoactivated tracer as shown in Fig. 5, with white denoting zero concentration, black maximum concentration $(c=1)$. The width of the pulse is approximately $400 \mu \mathrm{m}$. For boundary conditions, we assume that the flux of tracer is zero as $x \rightarrow \pm \infty$-solving numerically on a finite domain we must approximate this by $\partial c / \partial x=0$ at the edges $x=0$ and $x=X$. We also assume that no tracer is absorbed by the epithelium, so that $\partial c / \partial z=0$ on $z=0$, and that there is no flux through the free surface. For a flat free surface, this is simply $\partial c / \partial z=0$ on $z=H$.

As discussed above, the diffusion coefficient in the PCL was found to be around $D_{P}=160 \mu \mathrm{m}^{2} / \mathrm{s}$, in the mucous layer to be $D_{M}=3.6 \mu \mathrm{m}^{2} / \mathrm{s}$. To begin with, we 

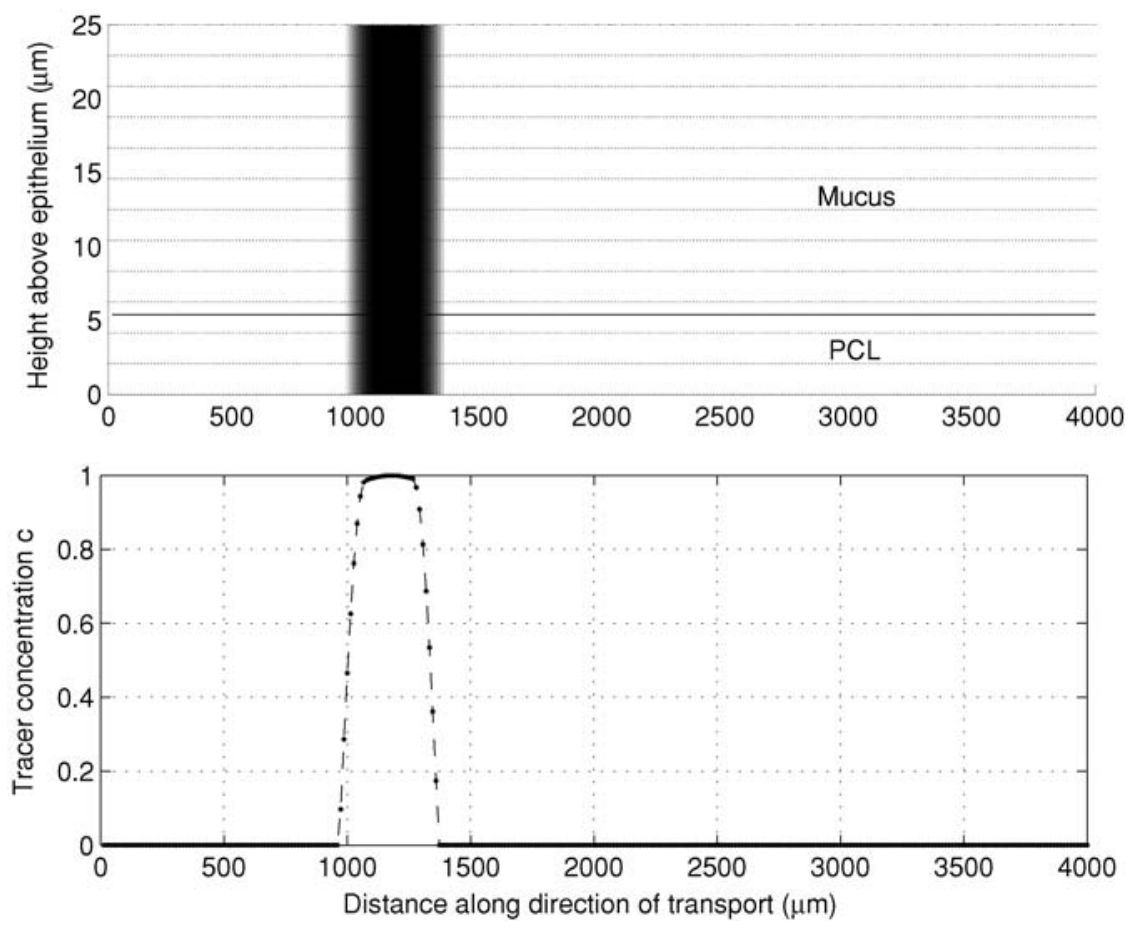

Fig. 5 Initial conditions - a tracer pulse with width approximately $400 \mu \mathrm{m}$.

model the variation of $D(z)$ in the ASL by the function

$$
D_{1}(z)=\frac{1}{2}\left(\left(D_{P}-D_{M}\right) \tanh \left(40\left[\frac{h-z}{L}\right]\right)+D_{P}+D_{M}\right),
$$

where $h=0.9 \mu \mathrm{m}, L=6 \mu \mathrm{m}$. This is equal to $D_{P}$ throughout most of the region $0<z<h$ and to $D_{M}$ throughout most of the region $h<z<H$, with a smooth transition in a region of width approximately $0.8 \mu \mathrm{m}$. The smooth transition means that the term $\mathrm{d} D / \mathrm{d} z$ may be determined in Eq. (5). The diffusion profile is shown in Fig. 6(A). In the following sections we describe the numerical solution method and results.

\section{Numerical solution}

An extension of the ADI method of Douglas, Peaceman and Rachford (see for example, Douglas and Peaceman, 1955) is used here, as in Blake and Gaffney (2001) due to its efficiency and stability. The scheme, originally for simulating diffusion of heat, is unconditionally stable, second order accurate in time and space, and at each time steps requires only the solution of a set of tridiagonal matrix equations. In order to discretise the advective terms, Blake and Gaffney (2001) used first order upwind discretisation. This scheme is more stable than central differencing, but 

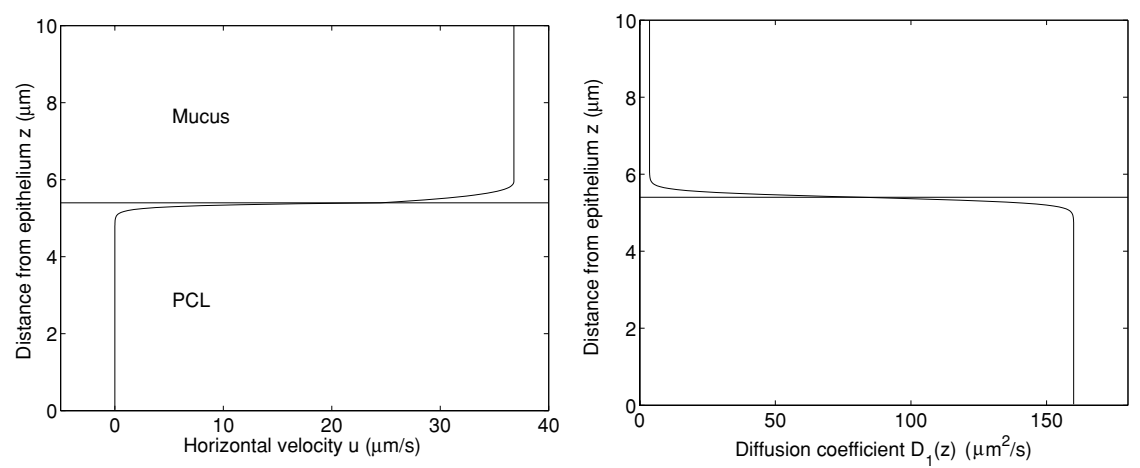

Fig. 6 A-mean horizontal velocity $u$ versus height above epithelium from Smith et al. (2006b). B-diffusion profile $D_{1}(z)$.

has the disadvantages of being only first order accurate and introducing numerical diffusion. Since additional diffusion would have the effect of reducing the separation between the tracer pulses in the mucus and PCL, this could produce misleading results. Instead we shall use the QUICK discretisation (Leonard, 1979). For 1D problems, QUICK is stable, formally third-order accurate, conservative and does not introduce numerical diffusion. The scheme does not have these formal properties in $2 \mathrm{D}$, but our results show greatly improved spatial convergence and very acceptably accurate conservation of $c$. Implementation is very straightforward, since at each timestep it is necessary to solve a set of pentadiagonal matrix equations, which can be done with comparable efficiency to tridiagonal system.

To compare and interpret the results of different simulations quantitatively, we calculate the distance moved by the centroid of the tracer pulse at different levels in the fluid:

$$
d(z, t):=\frac{\int_{x=-\infty}^{\infty} x c(x, z, t) \mathrm{d} x}{\int_{x=-\infty}^{\infty} c(x, z, t) \mathrm{d} x} .
$$

We then define the transport ratio $r(t)=d(H, t) / d(0, t)$, the ratio of the tracer transport at the top of the mucous layer to the bottom of the PCL. Due to the fact that vertical diffusion acts on a timescale of up to $12 \mathrm{~s}$, it is expected that $r(t)$ will not 'converge' to a final value until a simulation has been performed for a similar time period. Cotransport over the timescale of the experiment will be represented ideally by $r(t) \sim 1$ for $t=20 \mathrm{~s}$.

\section{Steady transport results}

\subsection{Steady profile of Smith et al. (2006b)}

We begin by presenting results for the steady velocity $\mathbf{u}=(u(z), 0)$ field shown in Fig. 6(B), and for the diffusion profile $D_{1}(z)$, shown in Fig. 6(A). The velocity 
Table 1 Numerical simulation results for Smith et al. (2006b) steady velocity profile (low mean PCL transport) and diffusion profile $D_{1}$

\begin{tabular}{lllllll}
\hline$t_{\text {out }}$ & $\delta x$ & $\delta z$ & $\delta t$ & $d\left(0, t_{\text {out }}\right)$ & $d\left(25, t_{\text {out }}\right)$ & $r\left(t_{\text {out }}\right)$ \\
\hline 5 & 6.6667 & 0.1250 & 0.02 & 74.920 & 191.28 & 2.5531 \\
10 & 6.6667 & 0.1250 & 0.02 & 182.28 & 380.19 & 2.0858 \\
20 & 6.6667 & 0.1250 & 0.02 & 431.19 & 736.86 & 1.7089 \\
30 & 6.6667 & 0.1250 & 0.02 & 703.34 & 1068.7 & 1.5194 \\
\hline
\end{tabular}

The separation between the pulses in the mucus and PCL continues to increase over the course of the simulation. All times in seconds, distances in $\mu \mathrm{m}$.

and diffusion profiles are shown together in Fig. 6 for comparison. A domain of width $0<x<4000 \mu \mathrm{m}$ and height $0<z<25 \mu \mathrm{m}$ was used. The ASL height was chosen to match the hTBE experiments, but does not appear to be critical to the results. Space and time steps of $\delta x=6.67 \mu \mathrm{m}, \delta z=0.125 \mu \mathrm{m}$ and $\delta t=0.02 \mathrm{~s}$ were used-we repeated the simulation with each of these parameters halved, showing that convergence in the transport ratio $r(t)$ was achieved to $2 \mathrm{~d}$. p.

Examining Table 1 , we see that $r(30) \approx 1.52$, meaning that the tracer centroid in the mucous layer has been transported $52 \%$ farther than the tracer centroid in the PCL. In the hTBE experiments, this would mean that the tracer pulse would smear along, as viewed from above, which is not what was observed. As found by Blake and Gaffney (2001) and Barlow (2000), tracer transport in the PCL is still much larger than would be estimated from advection alone, due to vertical diffusion. By examining the results over 5-30 s, it is clear that for steady fluid transport vertical diffusion requires at least $30 \mathrm{~s}$ in order to take full effect, since the distance between the peaks continues to increase every $10 \mathrm{~s}$.

\subsection{Other steady velocity profiles}

In Table 2, we give results for the other mean velocity profiles depicted in Fig. 2. The 'plane Couette' flow, given by $u(z) \propto z$ is the profile calculated from the mucous layer $L<y<H$ moving with constant velocity, with no sublayer resistance or pressure gradients, similar to the results of the simple model of Barlow (2000). The ad hoc 'boundary layer' flow, which we represent by $u \propto 2 z^{1 / 5}-z^{-2 / 5}$, is based on the profile suggested by Matsui et al. (1998) in order to explain their experimental

Table 2 Numerical simulation results for the steady velocity profiles depicted in Figs. 6 and 7

\begin{tabular}{lllll}
\hline$t_{\text {out }}$ & Profile type & $d\left(0, t_{\text {out }}\right)$ & $d\left(25, t_{\text {out }}\right)$ & $r\left(t_{\text {out }}\right)$ \\
\hline 30 & Steady Smith et al. & 703.34 & 1068.7 & 1.5194 \\
30 & Steady plane Couette & 898.73 & 1103.6 & 1.2280 \\
30 & Steady Matsui et al. & 1119.1 & 1142.8 & 1.0212 \\
\hline
\end{tabular}

Mesh spacings and timestep were $\delta x=6.67 \mu \mathrm{m}, \delta z=0.125 \mu \mathrm{m}$ and $\delta t=0.02 \mathrm{~s}$. All times in seconds, distances in $\mu \mathrm{m}$. 

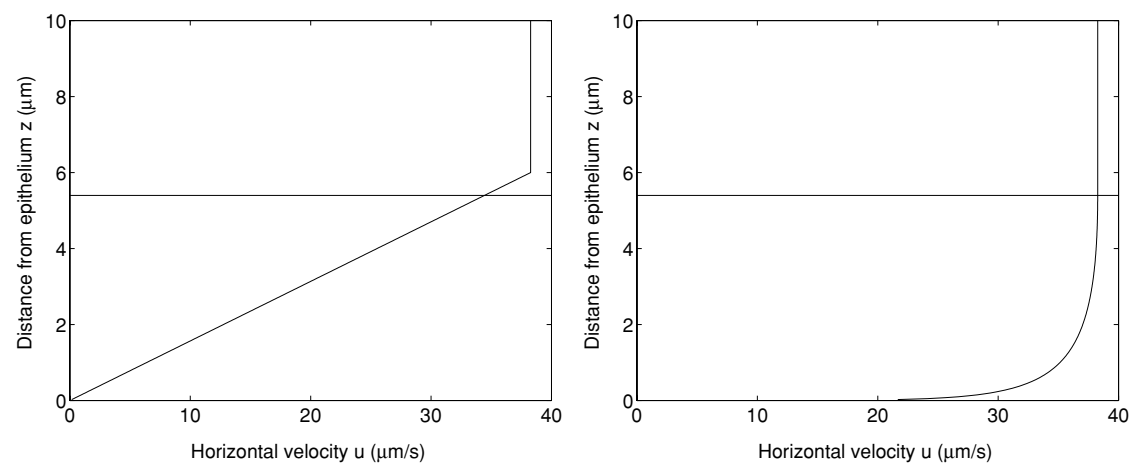

Fig. 7 Mean velocity profiles for 'shear driven' or plane Couette flow, and the 'boundary layer' flow, used for simulation. Profiles were taken from Fig. 2(B) and (C) respectively.

results, depicted in Fig. 2(C). Plots of the profiles used are given in Fig. 7. The simulation results are given in Fig. 8- the graphs show tracer concentration $c$ at the level of the epithelium $z=0$ and at the top of the mucus surface $z=25 \mu \mathrm{m}$. As might be expected, the plane Couette and boundary layer profiles cause successively greater transport of tracer in the PCL, with the boundary layer flow causing the pulses almost to match in size and position. It is interesting to note that for the low mean PCL transport results, the tracer in the mucous layer has an enlarged tail, showing the exchange of tracer between the two layers. This can also be visualised in the density plot of Fig. 9. The pulse in the PCL is very diffuse, due to the effect of horizontal diffusion. In the Couette and boundary layer graphs, the tracer pulse in the PCL is narrower, due to the fact that the pulses in the two layers are much more closely associated.

Quantitative measures of the results are shown in Table 2. Over the 30 s simulation period, only the 'boundary layer' flow provides a likely match with experimental results, the distance between the tracer centroids being only $23.7 \mu \mathrm{m}$. This is very much at variance with the results of our mechanical modelling-a considerable pressure gradient would be necessary to drive such a large flux of PCL through the dense field of cilia. In the next section, we examine the effects of introducing horizontal and vertical oscillation, and of altering the diffusion profile $D$.

\section{Oscillatory transport results}

\subsection{Oscillatory profile of Smith et al. (2006b)}

Simulations using oscillatory profiles calculated from the Fourier series from Smith et al. (2006b) and depicted in Fig. 4 are rather more computationally expensive, since a significant part of the algorithm involves calculating the matrix coefficients from the series $\sum_{n=0}^{15} \mathbf{u}_{n}$. To obtain results more efficiently we restrict the domain to $0<x<1800 \mu \mathrm{m}$ and $0<z<10 \mu \mathrm{m}$, and simulate dispersion for a shorter time period. The no-flux boundary condition is now applied at $z=10 \mu \mathrm{m}$. We have 

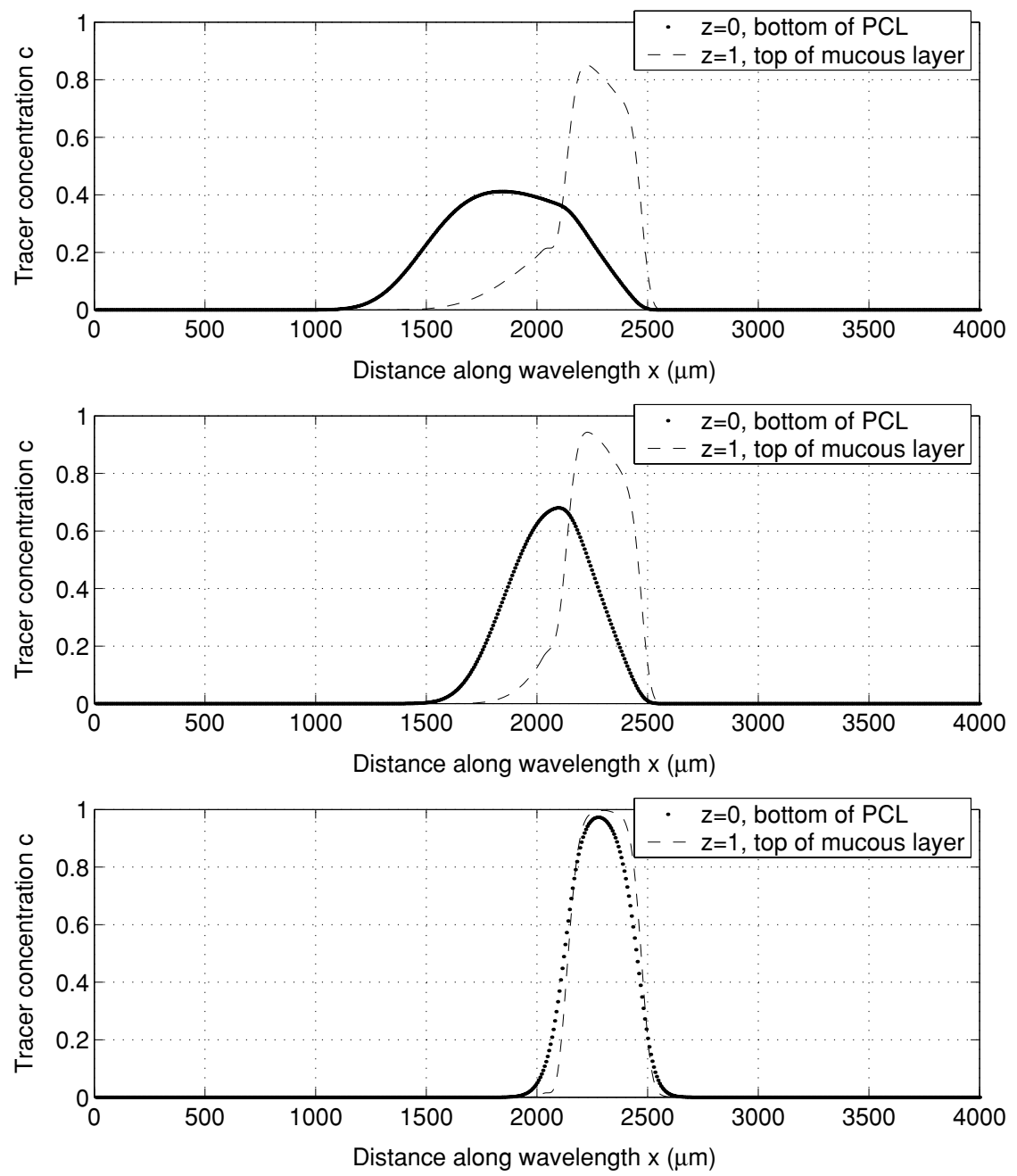

Fig. 8 Numerical simulation results - steady profiles with diffusion profile $D_{1}$. The traces show $c\left(x, z=0, t=t_{\mathrm{out}}\right)$ and $c\left(x, z=H, t=t_{\mathrm{out}}\right)$ as a function of $x$. Top-steady velocity from Smith et al. (2006b) (low mean PCL transport), as shown in Fig. 2(A). Middle-steady shear driven velocity (plane Couette flow), as shown in Fig. 2(B). Bottom-steady 'boundary layer' profile as predicted by Matsui et al. (1998) and shown in Fig. 2(C) (high mean PCL transport). Time duration $30 \mathrm{~s}$.

confirmed from simulations with the steady profile that the domain height does not have a significant effect. Efficiency is also greatly improved by employing an algorithm that exploits the periodicity of the ciliary beat, so that velocity values need only be calculated on a mesh covering one wavelength. For the oscillatory profiles the peak value of the velocity is of the order of 10 times that for the steady flow, so that the solution requires a finer spatial mesh. The timestep must also be reduced by a factor of ten in order to capture the detail of the ciliary beat 


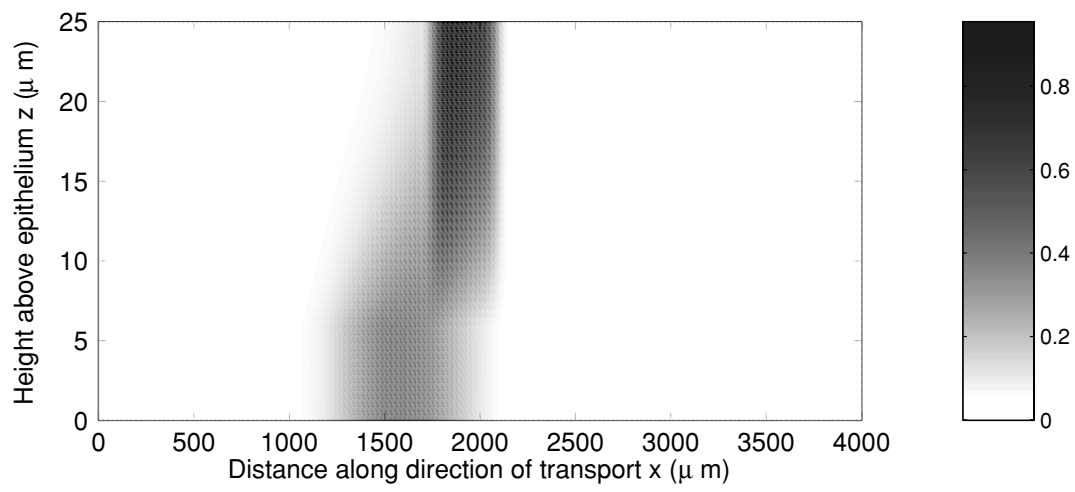

Fig. 9 Density plot of the tracer profile after 20 seconds of steady advection with the low mean PCL transport profile from Smith et al. (2006b).

cycle. A good estimate of the transport ratio can be determined using $\delta x=1.5 \mu \mathrm{m}$, $\delta z=0.0625 \mu \mathrm{m}$ and $\delta t=0.002 \mathrm{~s}$.

In Blake and Gaffney (2001) it was shown that an ad hoc oscillatory component substantially increased tracer transport in the PCL, although by how much was not given quantitatively. Table 3 shows the transport ratios for $t=5,10$ and $20 \mathrm{~s}$ for our mechanically derived oscillatory profile. After $20 \mathrm{~s}$, the transport ratio is only 1.18 , whereas for the steady profile was 1.70 . The addition of an oscillatory component hence makes a very significant difference. It was shown by Taylor (1953) that for flow in a narrow tube, shearing in the velocity field $\partial u / \partial z$ effectively increased the diffusivity of $c$. The oscillatory terms introduce large transient values of $\partial u / \partial z$ in the region $h<z<L$, which greatly enhance diffusion between the mucous layer and PCL, hence reducing the timescale of diffusive mixing. The ad hoc oscillatory component used by Blake and Gaffney (2001) similarly possessed a sharp gradient for $z$ close to $h$, being proportional to $\sqrt{4(z / h)(1-z / h)}$. It is interesting to note that the distance separating the peaks does not significantly increase between 5 and $10 \mathrm{~s}$, increasing from $54.6 \mu \mathrm{m}$ to $56.2 \mu \mathrm{m}$. The presence of oscillatory mixing hence reduces the effective vertical diffusive timescale to less than $5 \mathrm{~s}$, which is rather different from the steady transport results of Table 1. These results are also be visualised in Fig. 11, which shows tracer concentration as a density plot for the

Table 3 Numerical simulation results for Smith et al. (2006b) oscillatory velocity profile and diffusion profile $D_{1}$

\begin{tabular}{lllllll}
\hline$t_{\text {out }}$ & $\delta x$ & $\delta z$ & $\delta t$ & $d\left(0, t_{\text {out }}\right)$ & $d\left(10, t_{\text {out }}\right)$ & $r\left(t_{\text {out }}\right)$ \\
\hline 5 & 1.5 & 0.0625 & 0.002 & 67.983 & 122.61 & 1.8035 \\
10 & 1.5 & 0.0625 & 0.002 & 151.95 & 208.19 & 1.3702 \\
20 & 1.5 & 0.0625 & 0.002 & 320.75 & 377.04 & 1.1755 \\
\hline
\end{tabular}

The separation between the two pulses does not significantly increase after 5 seconds. All times in seconds, distances in $\mu \mathrm{m}$. 

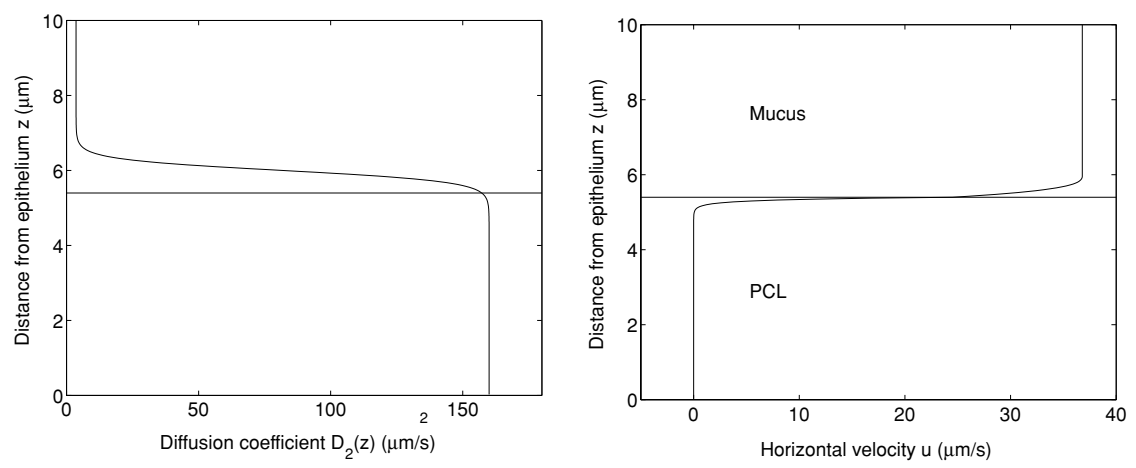

Fig. 10 Adjusted diffusion profile $D_{2}(z)$. Mean horizontal velocity $u$ versus height above epithelium from Smith et al. (2006b).

simulation with the oscillatory velocity field of Smith et al. (2006b) and diffusion profile $D_{1}(z)$.

The inclusion of oscillations has brought us considerably closer to the experimentally observed results. In the following sections we examine other effects which increase mixing further.

\subsection{Altering the diffusion profile}

The diffusion profile $D_{1}$ chosen for the above simulations decreased abruptly from $160 \mu \mathrm{m}^{2} / \mathrm{s}$ to $3.6 \mu \mathrm{m}^{2} / \mathrm{s}$ at the mucus-PCL interface $y=h$. However, it is likely that vertical mixing due to penetration of the mucous layer by cilia, which was not a feature of the Smith et al. model, may substantially enhance vertical 'diffusion'. A simple phenomenological model of this advective mixing is to increase the diffusion coefficient in this region by moving the transition in $D(z)$ further up into the mucous layer, and smoothing the transition, so that

$$
D_{2}(z)=\frac{1}{2}\left(\left(D_{P}-D_{M}\right) \tanh \left(20\left[\frac{L-z}{L}\right]\right)+D_{P}+D_{M}\right) .
$$

The velocity profile and diffusion profile $D_{2}(z)$ are shown in Fig. 10. The results of $20 \mathrm{~s}$ simulation are shown in Table 4 and Fig. 13, middle graph. The pulses separate by just $35.9 \mu \mathrm{m}$ after $20 \mathrm{~s}$, a $10.7 \%$ ratio compared with the $17.6 \%$ ratio observed for $D_{1}$.

\subsection{Mean profile of Fulford and Blake (1986) with oscillations}

As discussed in Section 3, although the Smith et al. model produces a mean profile superficially very similar to the results of Fulford and Blake (1986), the earlier model predicted significant fluid transport in the upper part of the PCL, likely due to the fact that the cilia bend closer to the epithelium during the recovery stroke and so do not resist the flow of fluid. Recent detailed modelling of the flow in the 


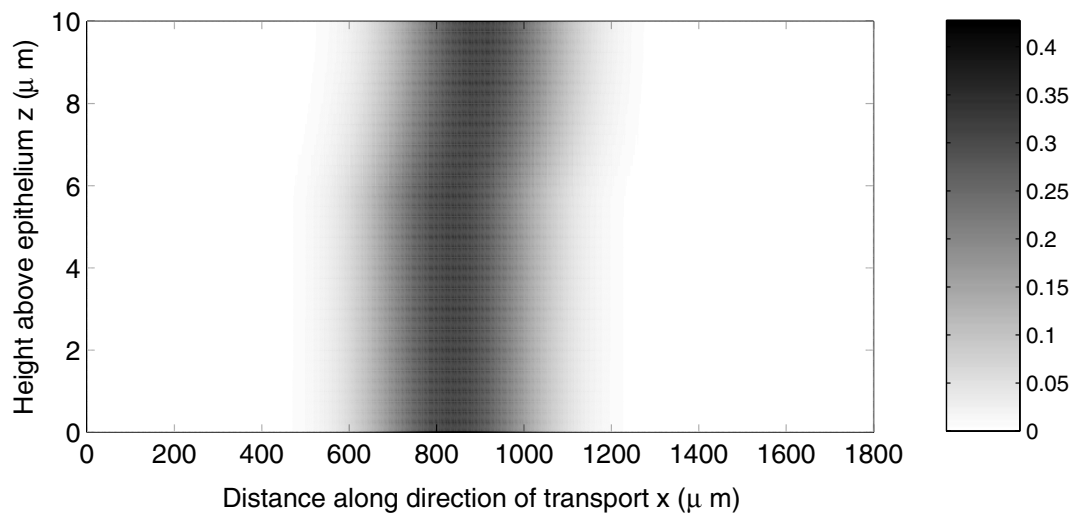

Fig. 11 Density plot of the tracer profile after 20 seconds of oscillatory advection with the velocity field from Smith et al. (2006b). If comparing with Fig. 9, note that a smaller domain is used for the oscillatory simulations since this does not significantly affect separation of the pulses.

PCL considering the cilia as discrete objects (Smith et al., 2006a) confirms thisthere is continuous positive fluid transport above the cilia tips during the recovery part of the beat cycle. A qualitative representation of this type of profile may be obtained by setting $h=0.8 L$ for the steady velocity component of the traction layer model. We then have the profile shown in Fig. 12, with the standard traction layer profile for comparison. Examining the numerical simulation results for this profile, shown in Table 4, the separation after $20 \mathrm{~s}$ is $51.6 \mu \mathrm{m}$, a little less than the $56.3 \mu \mathrm{m}$ observed for the standard profile, with $r(20)=1.14$. Combining a Fulford and Blake (1986) type profile with the diffusion coefficient $D_{2}$ produces still less separation-33.0 $\mu \mathrm{m}$, with $r(20)=1.09$.

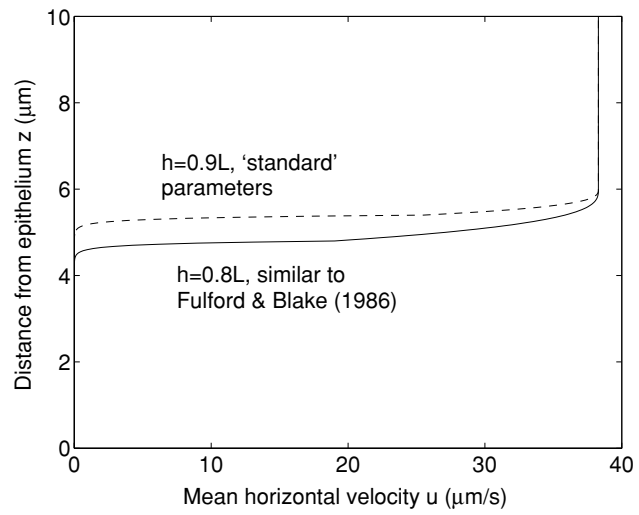

Fig. 12 (Solid line) traction layer mean profile with $h=0.8 L$, representative of mean velocity from Fulford and Blake (1986), showing greater fluid transport in the upper part of the PCL. (Dotted line) mean velocity with $h=0.9 L$ for comparison. 

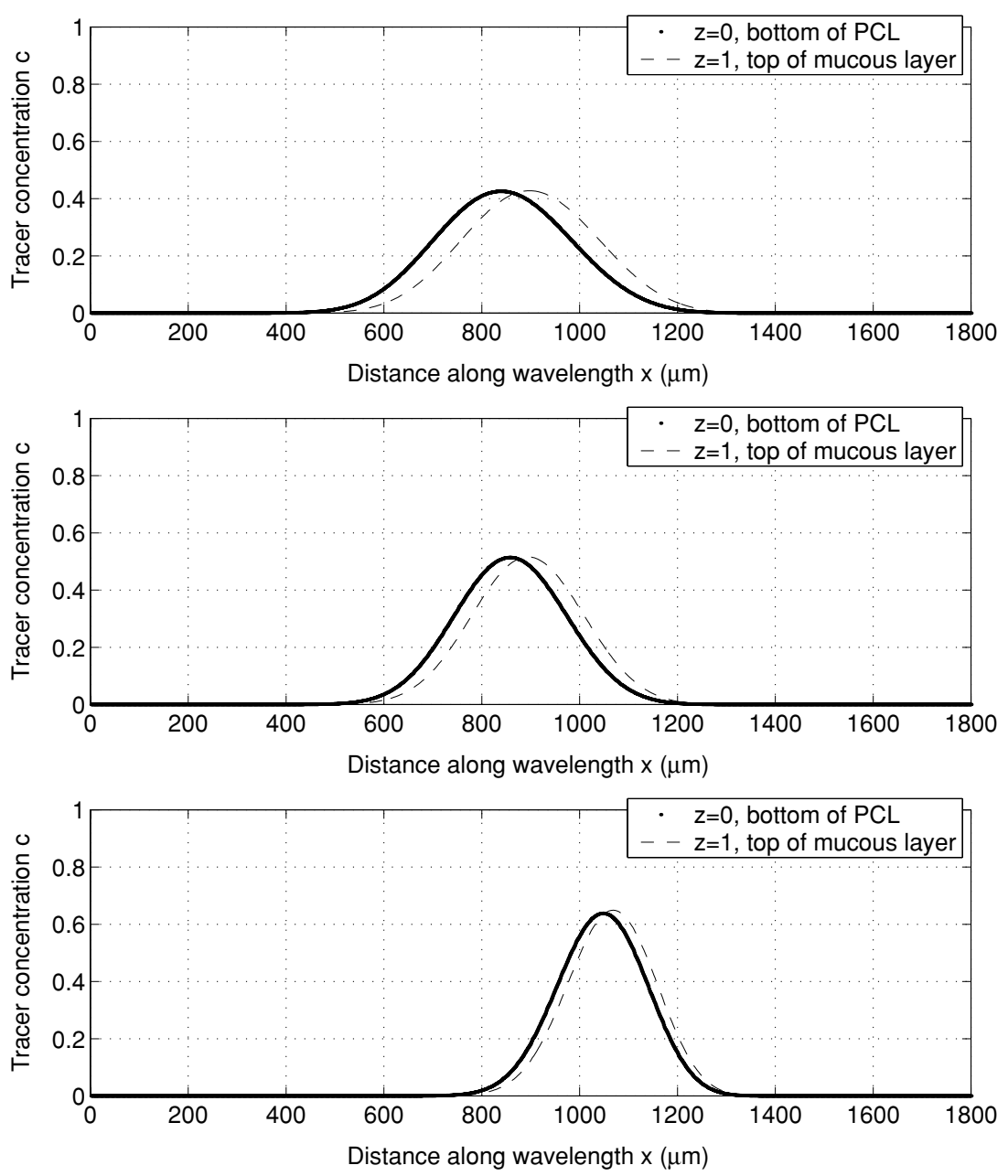

Fig. 13 Numerical simulation results—oscillatory profiles. Top—oscillatory velocity from Smith et al. (2006b) (low mean PCL transport), with diffusion $D_{1}(z)$. Middle—oscillatory velocity from Smith et al. (2006b) (low mean PCL transport), with diffusion $D_{2}(z)$. Bottom—ad hoc oscillatory velocity with plane Couette steady flow (medium mean PCL transport) and diffusion $D_{2}(z)$. The bottom profile shows greater transport than the other two due to the greater fluid flux in the velocity term. Time duration $20 \mathrm{~s}$.

\subsection{Ad hoc 'plane Couette' oscillatory profile}

In order to examine the effect of greater PCL transport with physically realistic oscillations, we combine the plane Couette profile shown in Fig. 7 with the timedependent terms $\mathbf{u}_{1}, \ldots, \mathbf{u}_{15}$ from the traction layer model. We simulated dispersion with the adjusted diffusion profile $D_{2}(z)$, again the results are shown in Table 4 and Fig. 13, bottom graph. After $20 \mathrm{~s}$, the separation between the two layers is just $3.9 \%$. This is likely to be consistent with the results of Matsui et al. (1998), 
Table 4 Numerical simulation results for various oscillatory profiles and diffusion coefficients $D_{1}(z)$ and $D_{2}(z)$

\begin{tabular}{llllll}
\hline$t_{\text {out }}$ & $D(z)$ & Velocity field $\mathbf{u}$ & $d\left(0, t_{\text {out }}\right)$ & $d\left(10, t_{\text {out }}\right)$ & $r\left(t_{\text {out }}\right)$ \\
\hline 20 & $D_{1}(z)$ & Traction layer & 320.75 & 377.04 & 1.1755 \\
20 & $D_{2}(z)$ & Traction layer & 334.98 & 370.90 & 1.1072 \\
20 & $D_{1}(z)$ & Oscillatory Fulford and Blake & 359.76 & 411.37 & 1.1435 \\
20 & $D_{2}(z)$ & Oscillatory Fulford and Blake & 372.84 & 405.82 & 1.0885 \\
20 & $D_{2}(z)$ & Oscillatory Couette & 520.04 & 540.48 & 1.0393 \\
20 & $D_{1}(z)$ & Oscillatory Matsui et al. & 728.13 & 732.00 & 1.0053 \\
\hline
\end{tabular}

All times in seconds, distances in $\mu \mathrm{m}$.

since a $3.9 \%$ separation between the pulses is unlikely to be apparent in the images produced. Hence we see that the Matsui et al. profile 2(C) is not a necessary consequence of their observations-only 50\% PCL transport is necessary to give apparent cotransport of tracer between the layers. It should be realised that this does not disprove the suggested profile of Matsui et al. (1998), and indeed in the presence of osmotic pressure gradients, it has not been shown that such a flow could not occur.

The tracer pulses predicted by the traction layer and Couette simulations are shown in Fig. 13. As for Fig. 8, it is interesting to note that when the pulses separate by less, the horizontal diffusion is reduced in both layers. The most striking comparision is between the top and middle graphs of Fig. 13, which depict results differing only in the diffusion coefficient $D(z)$. The middle graph, corresponding to greater diffusion at the level of the interface, actually shows less horizontal spread than the top graph. This is due to the fact that increased separation of the tracer pulse in the $D_{1}$ simulation results in non-zero gradients $\partial c / \partial z$ over a wider region and hence greater vertical diffusion between the layers. This results in apparent greater horizontal diffusion, even though $D_{1} \leqslant D_{2}$. We remark that the effect of vertical diffusion causing increased horizontal spreading is similar to the mechanism responsible for Taylor disperson. The fact that in experiments there was not significant horizontal spreading of the tracer pulses certainly argues for any separation between the two layers being small.

Finally, we note that for the suggested profile of Matsui et al., when combined with oscillations, leads to a separation of just $0.5 \%$, as might be expected.

\section{Conclusions}

This study builds on the existing knowledge that diffusion in the narrow ASL substantially alters concentration gradients of advecting tracers (Taylor, 1951; Blake and Gaffney, 2001; Mitran, 2004), by simulating the combined effect of diffusion and mechanically derived oscillations, using the QUICK advective scheme which does not introduce potentially misleading numerical diffusion. From our results it is now easier to reconcile the original experimental results of Matsui et al. (1998) with theoretical modelling work, even without considering flow due to osmotic pressure gradients. By including the oscillatory flow of Smith et al. (2006b), even 
with very low mean transport of PCL, we predicted results with relatively little 'smearing' of the tracer pulses. Adding a diffusive term to represent ciliary mixing at the interface leads to even closer tracer transport, with the tracer centroids separating by only $10.7 \%$ after $20 \mathrm{~s}$. Increasing transport in the upper part of the PCL reduces the separation further to $8.85 \%$, and refining our fluid dynamical model of the PCL in order to quantify this effect should be a subject for future research. Consideration of the combined effect of diffusion and cilia oscillation will be important other biological systems, for example morphogen transport in the embryonic node (Okada et al., 2005) and chemical attractant dispersion in the female reproductive tract (Fauci and Dillon, 2006).

However, we noted from our simulations that even a very small separation between the pulses would result in considerably increased horizontal diffusion, unlike that which was observed by Matsui et al. (1998). We can deduce from this that the tracer pulses in the experiment must have been very closely associated. Hence it appears likely that there is both greater fluid transport than predicted by the traction layer model, and there may be additional effects such as vertical mixing near the interface. Future work should address both of these issues, for instance by extending the traction layer model.

Finally, it is clear that the mean fluid transport in the PCL need not be anywhere near as large as originally suggested by Matsui et al. (1998). The simulation with the plane Couette mean profile together with oscillations predicted only $3.9 \%$ separation between the pulses, hence we concluded that PCL transport need not be any larger than $50 \%$ of that of mucus. It remains to be determined whether osmotic flows could produce such large PCL transport, and this too should be a subject for future work. In summary:

(i) In the presence of oscillations, even with very small mean PCL transport, the tracer pulses in the two layers transport at similar speeds, differing only by around $18 \%$.

(ii) Adding physically reasonable transport in the upper part of the PCL, and simulated 'mixing' at the interface, reduces this difference to less than $9 \%$.

(iii) The lack of horizontal spreading in experiment suggests that the tracer pulses were still more closely associated.

(iv) If the PCL transport is only $50 \%$ of the mucus transport, the tracer pulses will stay very closely associated, to within $4 \%$.

\section{Acknowledgements}

The authors would like to thank faculty and students at the Cystic Fibrosis/Pulmonary Research and Treatment Center and the Department of Applied Mathematics, University of North Carolina, Chapel Hill for valuable discussions, and in particular for allowing DJS to see the hTBE cultures first hand. DJS would also like to thank the EPSRC for financial support. 


\section{References}

Barlow, E., 2000. Modelling Muco-Ciliary Transport in the Lung. Master's Thesis, School of Mathematics and Statistics, University of Birmingham, Birmingham, UK.

Blake, J.R., Gaffney, E.A., 2001. Modeling aspects of tracer transport in mucociliary flows. In: Salathe, M. (Ed.), Cilia and Mucus: From Development to Respiratory Defense. Marcel Dekker, New York, pp. 291-302.

Boucher, R.C., 1994, Human airway ion transport. Part 1. Am. J. Respir. Crit. Care Med. 150, 271-281.

Douglas, J., Jr., Peaceman, D., 1955. Numerical solution of two-dimensional heat flow problems. A. I. Chem. E. J. 1, 505-512.

Fauci, L., Dillon, R., 2006. Biofluidmechanics of reproduction. Ann. Rev. Fluid Mech. 38(1), 371394.

Fulford, G.R., Blake, J.R., 1986. Muco-ciliary transport in the lung. J. Theor. Biol. 121, 381-402.

Leonard, B.P., 1979. A stable and accurate convective modelling procedure based on quadratic upstream interpolation. Comput. Methods Appl. Mech. Eng. 19, 459-98.

Lutz, R.J., Litt, M., Charkrin, L.W., 1973. Physical-chemical factors in mucus rheology. In: Gabelnick, H.L., Litt, M. (Eds.), Rheology of Biological Systems. Charles C. Thomas, Springfield, IL, pp. 119-157.

Matsui, H., Randell, S.H., Peretti, S.W., Davis, C.W., Boucher, R.C., 1998. Coordinated clearance of periciliary liquid and mucus from airway surfaces. J. Clin. Invest. 102(6), 1125-1131.

Mitran, S., 2004. Computation of advection and diffusion of a passive tracer in a prescribed velocity field. Available: http://www.amath.unc.edu/Faculty/mitran/research/PCL.html.

Okada, Y., Takeda, S., Tanaka, Y., Izpisúa Belmonte, J.-C., Hirokawa, N., 2005. Mechanism of nodal flow: A conserved symmetry breaking event in left-right axis determination. Cell 121, 633-644.

Quinton, P.M., 1994. Viscosity versus composition in airway pathology (editorial). Am. J. Respir. Crit. Care Med. 149, 6-7.

Sanderson, M.J., Sleigh, M.A., 1981. Ciliary activity of cultured rabbit tracheal epithelium: Beat pattern and metachrony. J. Cell Sci. 47, 331-341.

Sleigh, M.A., Blake, J.R., Liron, N., 1988. The propulsion of mucus by cilia. Am. Rev. Respir. Dis. 137, 726-741.

Smith, D.J., Gaffney, E.A., Blake, J.R., 2006a. Discrete cilia modelling with singularity distributions: Application to the embryonic node and the airway surface liquid. To appear in Bull. Math. Biol.

Smith, D.J., Gaffney, E.A., Blake, J.R., 2006b. A viscoelastic traction layer model of muco-ciliary transport. Published online first in Bull. Math. Biol. with doi: 10.1007/s11538-005-9036-x

Smith, J.J., Travis, S.M., Greenberg, E.P., Welsh, M.J., 1996. Cystic fibrosis airway epithelia fail to kill bacteria because of abnormal airway surface liquid. Cell 85, 229-236.

Taylor, G.I., 1951. Analysis of the swimming of microscopic organisms. Proc. R. Soc. Lond. A209, $447-461$.

Taylor, G.I., 1953. Dispersion of soluble matter in solvent flowing slowly throught a tube. Proc. R. Soc. Lond. A219, 186-203.

Toskala, E., 1994. Normal and Pathological Respiratory Cilia. An Experimental and Clinical Study With Special Reference to Scanning Electron Microscopy. PhD Thesis, University of Kuopio, Finland. 